\title{
The Family of Carabidae (Coleoptera) in Artvin Hatila National Park of Turkey
}

\author{
Temel Gokturk ${ }^{1 *}$, Alim Celik ${ }^{2}$ \\ ${ }^{1 *}$ Artvin Coruh University, Faculty of Forestry, Department of Forest Entomology and Protection, Artvin, Turkey \\ ${ }^{2}$ Artvin Forest Department, Forest Protection, Artvin, Turkey
}

\begin{abstract}
The faunistical studies on the family Carabidae (Coleoptera) species in Artvin Hatila National Park in Turkey. In this study, totally 32 species belonging to Pterostichinae, Brachininae, Carabinae, Platyninae, Lebiinae, Nebriinae and Harpalinae subfamilies of Carabidae were collected from Artvin province during 2011-2014. Among these, Amara lucida Duftschmid, Amara aulica Panzer, Brachinus elegans Chaudoir, Brachinus crepitans Linné, Carabus scabrosus Olivier, Anisodactylus binotatus Fabricius, Carabus coriaceus Linnaeus, Carabus mulsantianus Paykull, Carabus graecus Dejean, Harpalus affinis Schrank, Harpalus caspius Panzer, Ophonus cribricollis Dejean, Ophonus azureus Fabricius, Ophonus subquadratus Dejean, Pterostichus anthracinum Illiger are the first records from Artvin.
\end{abstract}

Keywords - Carabidae, Artvin Hatila National Park.

\section{INTRODUCTION}

The family of Carabidae is one of the biggest family of the Coleoptera order in terms of the number of species as about 40.000 in the worldwide [1]. The species of the Carabidae family lives under the stones, logs and crusts [2]. $73,5 \%$ of these species lives carnivorously, $19,5 \%$ of them lives omnivorous and $8,1 \%$ of them lives fitofag [2]. The carnivore Carabides are usually eat insects, earthworms, snails, caterpillars and tube lights $[3,4]$; and fitofags eats plants [5,6]. Some carnivore species of carabids are used significant elements of the biological contention [7]. Carabidae where they live are one of the best bioindicators for the protection of ecosystems $[8,9,10,11]$.

The Carabidae family is pullulated with the species and more than 170 species have been determined in the based on 1100 species in Turkey. Also $41 \%$ of them are endemic. This number does not reflect the actual number of the Carabidae species in Turkey [12,13,14].

There are several studies about the family of Carabidae in Turkey $\quad[12,15,16,17,18,19,20,21,22,23,24,25,26,27,28$, $29,30,31,32,33]$. But there has not been conducted any study about the Carabidae fauna of Artvin. It is aimed that this research define the fauna of Carabidiae family in
Hatila Valley National Park and around by using of the pitfall trap method.

\section{MATERIAL AND METHODS}

Artvin Hatila Valley National Park was announced as a national park in 1994 and became one of the 40 national parks in our country. This national park area has 16998hectare area and the GPS coordinates of $41^{\circ} 11^{\prime} 11^{\prime \prime} \mathrm{N}, 41^{\circ}$ $44^{\prime} 09^{\prime} \mathrm{E}^{\prime}$. The altitude of the highest point is $3224 \mathrm{~m}$ (Kurt Mountain), and the lowest point is $160 \mathrm{~m}$ (Çoruh River). This national park area has relict and endemic plant cover, unusual geological and geomorphological structure, unique scenic beauty, rich fauna and recreational potential. Semiarid, subhumid and humid climates are seen in the area. According to the grid system that was applied by Davis that this area in A8 square and Colchis part of EuroSiberian flora area of Holarctic flora region concerning the phytogeography and flora areas [34]. The field of the study has characteristics of a transition region between Black Sea climate and Continental climate [35].

The samples were collected from the Artvin Hatila National Park in between 2011-2014 years constitute the primary material of the study. Other materials are as follow; pitfall traps for collecting the species, pliers, stereomicroscope, numbered insect stings, glass materials and various chemicals for preparation. The samples of the species belong to Carabidae family were collected from the weeds (meadow grass, ferns, etc.) unopened areas for agriculture and forest lands.

The most of the species of Carabidae lives under the soil and several studies show that the pitfall traps were used to catch them in several studies. 80 pitfall traps were placed in 20 different areas by embedding till on the ground level. The traps has plastic containers were places and specially prepared malt solution and land snails belong to the families of Cochlostomatidae, Pomatiidae, Truncatellidae, Aciculidae. These traps were controlled every 15 daysand insects were collected from their in and the malt solution and snails were changed as well. The malt solution was prepared by boiling one litter beer and 250g sugar together, then refrigerated. The preparation was applied to the Carabidae species in the laboratory conditions. The 
diagnosis of these collected samples was actualized by using the determination keys of Lindroth [36], Geigenmüller \& Trautner [37], Forsythe [38] and Hurka [1].

The similar samples were recognized in the forest entomology laboratary in Artvin Çoruh University After that some species were determineted Protection Department Entomology Laboratory and Entomology Museum of Plant Protection of Agricultural Faculty of Erzurum Atatürk University. The studies about Carabidae species were useful for publishing Lindroth [36], Lodos [12], Gueorguiev \& Gueorguiev [37], Kryzanovsky et al., [39], Hurka [1], Casale and Taglianti [40], Neculiseanu and Matalin [41], Casale et al., [13] and Kesdek \& Yildırım [42] the distribution of species in Turkey and the world.

\section{RESULTS AND DISCUSSION}

As a result of the studies, 32 Carabidae species belonging to Pterostichinae (9), Brachininae (2), Carabinae (14), Platyninae (1), Lebiinae (1), Nebriinae (1), Harpalinae (4) 15 subspecies and 7 subfamilies were recorded in Hatila National Park in Artvin.

\section{Pterostichinae}

\section{Amara Bonelli, 1810}

\section{Amara similata (Gyllenhal, 1810)}

Material examined: Artvin - Hatila National Park:

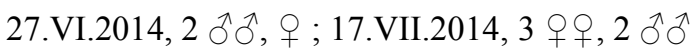

Distribution in Turkey: Artvin, İzmir, Kars [28,40].

Distribution in the world: Algeria, Anatolia, Asia, Caucasus Crimea, Czech Republic, Europe, Moldova, North Africa, Northwest Himalaya, Russia, Siberia, Slovakia, South of Armenia, Turkestan, Turkey, Ukraine $[1,37,39,40,41,43]$.

\section{Amara (Paracelia) saxicola (Zimmermann, 1831)}

Material examined: Artvin - Hatila National Park:

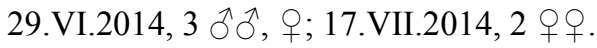

Distribution in Turkey: Artvin, Erzurum, Kars [40,44].

Distribution in the world: Anatolia, Armenia, Azerbaijan, Georgia, Caucasus and Mediterranean Countries, Crimean Mountains, Kazakhstan, Moldova, Southeast of Central Asia, Turkey, Ukraine [39,40,41].

\section{Amara aenea (De Geer, 1774)}

Material examined: Artvin - Hatila National Park:

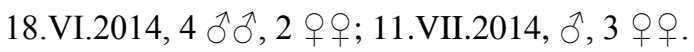

Distribution in Turkey: Ankara, Antalya, Ardahan, Artvin, Bitlis, Bolu, Erzincan, Eskişehir, Erzurum, Giresun, Gümüşhane, Iğdır, İzmir, Kars, Kastamonu, Konya, Manisa, Muğla, Nevşehir, Ordu, Rize, Sinop, Tokat $[23,29,40,43,45,46]$.
Distribution in the world: Armenia, Bosnia and Herzegovina, Bulgaria, Canary Islands, Corsica, Croatia, Cyprus, Czech Republic, Denmark, Estonia, Finland, France, Georgia, Greece, Jordan, Netherlands, Iraq, Iran, Ireland, Israel, Italy, Latvia, Liechtenstein, Lithuania, Lebanon, Luxembourg, Macedonia, Malta, Moldova, Nearikovo North Africa, Norway, Portugal, Romania, Russia, Sardinia, Slovakia, Slovenia, Spain, Sweden, Switzerland, Syria, Turkey, Ukraine, United Kingdom, Yugoslavia [46].

\section{Amara lucida (Duftschmid, 1812)}

Material examined: Artvin - Hatila National Park:

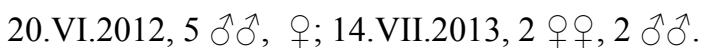

Distribution in Turkey: Anatolia, Erzurum, Kahramanmaraş [20,40,44].

Distribution in the world: Armenia, Austria, Azerbaijan, Belarus, Bosnia and Herzegovina, Bulgaria, Caucasus, Czech Republic, Denmark, Estonia, Finland, France, Georgia, Germany, Greece, Hungary, Ireland, Italy, Latvia, Lithuania, Luxembourg, Macedonia, Moldova, Montenegro, Moravia, Netherlands, North Africa, Norway, Poland, Portugal, Romania, Russia, Serbia, Slovakia, Slovenia, Transcaucasia, Ukraine [1,6,37,39].

\section{Amara ovata (Fabricius, 1792)}

Material examined: Artvin - Hatila National Park: 20.VI.2012, 5 ふふં; 14.VII.2013, 3 우, 2 ふす

Distribution in Turkey: Artvin, Erzurum, Kars [20,44].

Distribution in the world: Algeria, Austria, Azerbaijan, Belgium, Belarus, Bosnia and Herzegovina, Bulgaria, Caucasia, Czech Republic, Denmark, Estonia, Finland, France, Germany, Greece, Germany, Japan, Italy, Kazakhstan, Kyrgyzstan, Latvia, Lithuania, Luxembourg, Hungary, Macedonia, Moldova, Norway, Poland, Portugal, Romania, Russia, Serbia, Slovenia, Slovakia, Sweden, Switzerland, Syria, Tajikistan, Transcaucasia, Transbaikalia, Turkey, Turkmenistan, Ukraine, Urals, Uzbekistan [1,6,37,39].

\section{Amara (Curtonotus) aulica (Panzer, 1796)}

Material examined: Artvin - Hatila National Park: 28.VI.2013, 2 ふぶ; 10.VII.2014, ; 24.VII.2014, ․ .

Distribution in Turkey: Kahramanmaraş [20,40].

Distribution in the world: Albania, Armenia, Austria, Azerbaijan, Bosnia and Herzegovina, Canary Islands, Czech Republic, Denmark, Estonia, Faroe Islands, Finland, France, Georgia, Germany, Greece, Hungary, Ireland, Italy, Kazakhstan, Kyrgyzstan, Latvia, Lithuania, Macedonia, Mongolia, Moldova, Netherlands, North America, Montenegro, Norway, Poland, Romania, Russia, Serbia, Siberia, Slovakia, Tajikistan, Turkey, Turkmenistan, Ukraine, Uzbekistan [1,6,47]. 
Pterostichus Bonelli, 1810

Pterostichus (Melanius) anthracinum (Illiger, 1798)

Material examined: Artvin - Hatila National Park:

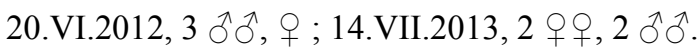

Distribution in Turkey: Anatolia (no locality) [40].

Distribution in the world: Europe, Bulgaria, Czech Republic, Iran, Kyrgyzstan, Moldova, Central Asia, Russia, Slovakia [1,37,39,40,41].

\section{Pterostichus (Melanius) nigrita (Paykull, 1790)}

Material examined: Artvin - Hatila National Park: 20.VI.2012, 2 ふふふ; 14.VII.2013, 3 우, Љ

Distribution in Turkey: Ardahan, Artvin, Eskişehir, Erzurum, Kars [21,40,42].

Distribution in the world: Bulgaria, Caucasus, Czech Republic, England, Central Asia and Europe, Moldova, Russia, Siberia Slovakia, Turkey, Turkistan $[1,37,39,40,41]$.

\section{Zabrus Clairville, 1806}

\section{Zabrus spinipes (Fabricius, 1798)}

Material examined: Hatila National Park: 30.VI.2014 §’; 22.VII.2014, 2 웅.

Distribution in Turkey: Ankara, Artvin, Trabzon [15,48].

Distribution in the world: Austria, Bulgaria, Czech Republic, Sweden, Hungary, Poland, Slovakia, Turkey, Greece, Yugoslavia [6].

\section{Brachininae}

\section{Brachinus Weber, 1801}

\section{Brachinus elegans (Chaudoir, 1842)}

Material examined: Artvin - Hatila National Park: 20.VI.2012, 3 우; $\widehat{\jmath}, 17$. III.2013, 2 우.

Distribution in Turkey: Tokat [48].

Distribution in the world: Armenia, Azerbaijan, Bosnia and Herzegovina, Bulgaria, Croatia, Czech Republic, France, Georgia, Iraq, Iran, Italy, Kazakhstan, Moldova, Morocco, Romania, Russia, Slovakia, Slovenia, Spain, Ukraine, Yugoslavia [6].

\section{Brachinus crepitans (Linné, 1758)}

Material examined: Artvin - Hatila National Park: 20.VI.2012, 2 ふふં; 14.VII.2013, 3 우, ふ.

Distribution in Turkey: Amasya, Giresun, Kahramanmaraş [20,40,48].

Distribution in the world: Azerbaijan, Belarus, Bosnia and Herzegovina, Croatia, Cyprus, Czech Republic, Denmark, Estonia, Finland, France, Georgia, Germany, Greece, Hungary, Luxembourg, Latvia, Moldova, Montenegro, Norway, Romania, Russia, Slovakia, Slovenia, Syria, Tajikistan, Turkmenistan, Ukraine, Yugoslavia [6].

\section{Carabinae}

Calosoma Weber, 1801

Calosoma inquisitor (Linnaeus, 1758)

Material examined: Artvin - Hatila National Park: 20

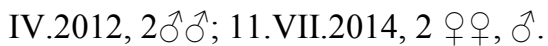

Distribution in Turkey: Artvin, Erzincan, Tunceli, Antalya [49].

Distribution in the world: Armenia, Azerbaijan, Belarus, Belgium, Germany, Greece, France, Hungary, Japan, Italy, Iran, Luxembourg, Morocco, Netherlands, North Africa, Norway, Poland, Russia, Slovakia, Slovenia, Sweden, Switzerland, Ukraine, United Kingdom, Turkey, [37].

\section{Calosoma sycophanta (Linné, 1758)}

Material examined: Artvin - Hatila National Park:

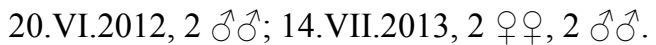

Distribution in Turkey: Ankara, Artvin, Adiyaman, Kahramanmaraş [15,20,40].

Distribution in the world: Albania, Armenia, Austria, Azerbaijan, Belgium, Bosnia and Herzegovina, Bulgaria, Czech Republic, China, Cyprus, Denmark, Estonia, Germany, Greece, France, Kazakhstan, Kyrgyzstan, Latvia, Lithuania, Macedonia, Moldova, Montenegro Morocco, South Moravia, Netherlands, Croatia, North Africa, North America, Poland, Portugal, Romania, Russia, Serbia, Siberia, Slovakia, Slovenia, Syria, Spain, Tunisia, Turkey, Turkmenistan, Ukraine, Uzbekistan $[1,6,37,39,47]$.

\section{Carabus Linnaeus, 1758 \\ Carabus convexus (Fabricius, 1775)}

Material examined: Artvin - Hatila National Park:

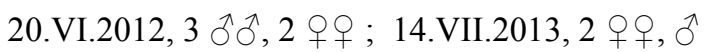

Distribution in Turkey: Black sea region of Turkey, Marmara Region, Rize [13,48].

Distribution in the world: Andorra, Austria, Belarus, Belgium, Bosnia and Herzegovina, Bulgaria, Denmark, Finland, France, Germany, Greece, Hungary, Italy, Kazakhstan, Luxembourg, Macedonia, Moldova, Norway, Netherlands, Poland, Romania, Russia, Slovakia, Slovenia, Spain, Sweden, Switzerland, Turkey, Ukraine, Yugoslavia [6].

\section{Carabus scabrosus (Olivier, 1795)}

Material examined: Artvin - Hatila National Park: 28.VI.2014, ふૈ, o; 07.VII.2014, 2 우.

Distribution in Turkey: Ordu [48].

Distribution in the world: Armenia, Azerbaijan, Bulgaria, Greece, Georgia, Iran, Turkey, Ukraine [6].

\section{Carabus (Procrustes) coriaceus (Linnaeus, 1758)}

Material examined: Artvin - Hatila National Park:

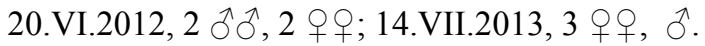


Distribution in Turkey: Izmir, Canakkale, Marmara Region, Muğla Western Mediterranean [13,29,46,49].

Distribution in the world: Armenia, Austria, Azerbaijan, Belarus, Bosnia and Herzegovina, Czech Republic, Denmark, Estonia, France, Georgia, Germany, Greece, Hungary, Iran, Iraq, Ireland, Israel, Italy, Jordan, Kazakhstan, Kyrgyzstan, Latvia, Lebanon, Lithuania, Luxembourg, Macedonia, Moldova, Netherlands, Norway, Poland, Romania, Russia, Sardinia, Sicily, Slovakia, Slovenia, Syria, Tajikistan, Turkey, Ukraine, Uzbekistan, Yugoslavia [13].

\section{Carabus mulsantianus (Paykull, 1790)}

Material examined: Artvin - Hatila National Park:

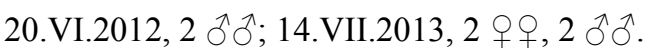

Distribution in Turkey: Kahramanmaras [15,50].

Distribution in the world: Turkey [6].

\section{Carabus (Pachystus) graecus (Dejean, 1826)}

Material examined: Artvin - Hatila National Park:

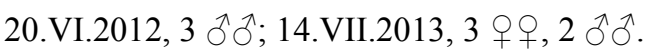

Distribution in Turkey: Aegean and Marmara Regions, Ankara, Canakkale, Western Mediterranean [15-50].

Distribution in the world: Armenia, Azerbaijan, Bulgaria, Caucasus Republics, Egypt, Greece, Georgia, Jordan, Iraq, Iran, Israel, Lebanon, Peninsula, Russia, Scandinavia, Syria, Turkey [13,51].

\section{Harpalus Latreille, 1802}

\section{Harpalus affinis (Schrank, 1781)}

Material examined: Artvin - Hatila National Park: 20.VI.2012, 4 ô; 14.VII.2013, 4 ㅇ.

Distribution in Turkey: Rize [48].

Distribution in the world: Albania, Andorra, Azerbaijan, Belarus, Bosnia and Herzegovina, Czech Republic, Denmark, Egypt, Estonia, Finland, France, Germany, Greece, Hungary, Iran, Ireland, Israel, Italy, Kazakhstan, Kyrgyzstan, Lichtenstein, Lithuania, Macedonia, Moldova, Mongolia, Netherlands, Norway, Poland, Portugal, Romania, Russia, Slovakia, Slovenia, Turkey, Ukraine, Yugoslavia, Sweden, Switzerland [6].

\section{Harpalus smaragdinus (Duftschmid, 1812)}

Material examined: Artvin - Hatila National Park:

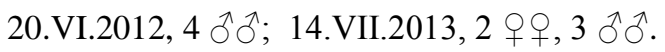

Distribution in Turkey: Antalya, Ardahan, Artvin, Bitlis, Bolu, Çanakkale, Çankırı, Erzurum, Giresun, Gümüşhane, İzmir, Kahramanmaraş, Kars, Malatya, Manisa, Sivas $[20,29,40,42,50]$.

Distribution in the world: Albania, Austria, Azerbaijan, Belarus, Bosnia and Herzegovina, Bulgaria, Caucasus, Central Asia, Crimea, Cyprus, Czech Republic, Denmark, Estonia, Finland, France, Georgia, Germany, Greece,
Hungary, Italy, Kazakhstan, Kyrgyzstan, Latvia, Lithuania, Luxembourg, Macedonia, Moldova, Montenegro, Norway, Poland, Romania, Russia, Serbia, Siberia, Slovakia, Slovenia, Sweden, Switzerland, Tajikistan, Turkey, Turkmenistan, Ukraine, United Kingdom, United States, Urals, Uzbekistan [1,6,37,39,47].

\section{Harpalus caspius (Panzer, 1797)}

Material examined: Artvin - Hatila National Park:

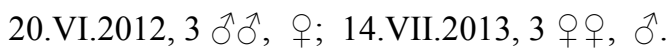

Distribution in Turkey: Anatolia (no locality) [39].

Distribution in the World: Armenia, Austria, Azerbaijan, Bulgaria, Caucasus, Crimea, Croatia, Czech Republic, Georgia, Germany, Hungary, Iran, Kazakhstan, Moldova, Montenegro, Slovakia, Slovenia, Turkey, Ukraine $[6,37,39]$.

\section{Harpalus distinguendus (Duftschmid, 1812)}

Material examined: Artvin - Hatila National Park: 30.VI.2013, 4 ऊิ ठึ.

Distribution in Turkey: Ankara, Antalya, Artvin, Aydın, Bergama, Bayburt, Burdur, Çanakkale, Erzincan, Erzurum, Giresun, Gümüşhane, Isparta, İçel, İstanbul, İzmir, Kahramanmaraş, Konya, Tokat, Trabzon [29,40,42,50].

Distribution in the world: Afghanistan, Azerbaijan, Caucasus, Central Asia, Czech Republic, Europe, Iran, Kazakhstan, Korea, Middle Mediterranean Countries, Mongolia, North East Africa, North East and West China, Northern Russia, Siberia, Slovakia [1,40,51].

\section{Poecilus Bonelli, 1810}

\section{Poecilus cupreus (Linnaeus, 1758)}

Material examined: Hatila National Park: 30.VI.2014, 4

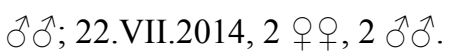

Distribution in Turkey: Ankara İzmir-Kemalpaşa, Antalya, Ardahan, Artvin, Aydın, Balıkesir, Bolu, Çanakkale, Çankırı, Erzurum, Kars, Kastamonu, Konya, Ordu, Sinop [29,40,50].

Distribution in the world: Albania, Armenia, Austria, Azerbaijan, Belarus, Belgium, Bosnia and Herzegovina, British Isles, Bulgaria, Caucasian Republics, Corsica, Crete, Croatia, Czech Republic, Denmark, Eastern Palearctic Region, Estonia, Finland, France, Georgia, Germany, Greece, Hungary, Iraq, Iran, Ireland, Israel, Italy, Jordan Latvia, Lebanon, Liechtenstein, Lithuania, Luxembourg, Macedonia, Moldova, Netherlands, Northern Ireland, Norway, Poland, Romania, Russia, Sardinia, Sicily, Slovakia, Slovenia, Spain, Sweden, Switzerland, Syria, the Arabian Peninsula, Turkey, Ukraine, Yugoslavia [51]. 
Pseudoophonus Motschulsky, 1844

Pseudoophonus rufipes (De Geer, 1774)

Material examined: Hatila National Park: 20.VI.2012, 2

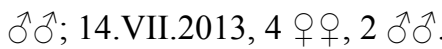

Distribution in Turkey: Adana, Adiyaman, Ankara, Antalya, Ardahan, Artvin, Bartın, Bingöl, Bursa, Çankırı, Diyarbakır, Erzincan, Erzurum, Eskişehir, Gaziantep, Giresun, Gümüşhane, Iğdır, Isparta, İçel, İzmir, Kahramanmaraş, Kayseri, Malatya, Karaman, Kars, Konya, Kütahya, Malatya, Muğla, Ordu, Osmaniye, Sivas, Tokat, Trabzon, Yalova [15,20,23,40,42,48,52,53].

Distribution in the world: Afghanistan, Albania, Albania, Austria, Azerbaijan, Algeria, Armenia, Belarus, Belgium, Bosnia and Herzegovina, Bulgaria, China, Crimea, Croatia, Czech Republic, Denmark, Egypt, Estonia, Finland, France, Georgia, Greece, Hungary, Ireland, Italy, Kazakhstan, Kyrgyzstan, Latvia, Lithuania, Luxembourg, Macedonia, Malta, Moldova, Morocco, Netherlands, North America, Norway, Poland, Portugal, Romania , Russia, Serbia, Siberia, Slovakia, Slovenia, Spain, Sweden, Switzerland, Tajikistan, Tunisia, Turkey, Turkmenistan, Ukraine, United Kingdom, Urals, Uzbekistan $[1,6,37,39,47,52]$.

\section{Pseudoophonus calceatus (Duftschmid, 1812)}

Material examined: Hatila National Park: 30.VI.2014, 2

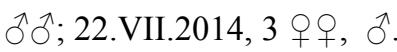

Distribution in Turkey: Artvin, Trabzon [48].

Distribution in the world: Armenia, Austria, Azerbaijan, Belgium, Bosnia and Herzegovina, Bulgaria, China, Croatia, Czech Republic, Denmark, Estonia, Finland, France, Greece, Georgia, Germany, Japan, Hungary, Kazakhstan, Kyrgyzstan, Latvia, Lithuania, Luxembourg, Macedonia, Moldova, Mongolia, Norway, North Korea, Poland, Portugal, Romania, Russia, Slovakia, Slovenia, Tajikistan, Turkmenistan, Ukraine, Uzbekistan, Yugoslavia [6].

\section{Platyninae}

\section{Dolichus Bonelli, 1810}

\section{Dolichus halensis (Schaller, 1783)}

Material examined: Hatila National Park: 30.VI.2014, 3 ふึે, q; 22.VII.2014, 4 우.

Distribution in Turkey: Ankara, Artvin [15,49].

Distribution in the world: Armenia, Caucasus, Central Asia, Central and South Russia, Kazakhstan, Siberia, Turkey, Ukraine $[39,40]$.

\section{Lebiinae}

Cymindis Latreille, 1806

Cymindis axillaris (Fabricius, 1794)

Material examined: Hatila National Park: 20.VI.2012, 2 ふふ઼; 14.VII.2013, ․
Distribution in Turkey: Artvin, Çorum, Kahramanmaraş [20,48].

Distribution in the world: Austria, Azerbaijan, Bosnia and Herzegovina, Cyprus, Czech Republic, Denmark, Egypt, France, Germany, Greece, Hungary, Ireland, Israel, Italy, Latvia, Lebanon, Libya, Lithuania, Luxembourg, Moldova, Norway, Poland, Portugal, Romania, Russia, Slovakia, Slovenia, Syria, Turkey, Turkmenistan, Ukraine, Ukraine, United Arab Emirates, United Kingdom, United States, Yugoslavia [6].

\section{Nebriinae}

Nebria Latreille, 1802

Nebria brevicollis (Fabricius, 1792)

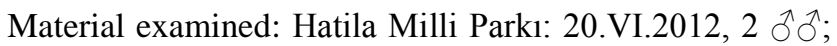
14.VII.2013, 3 우우,

Distribution in Turkey: Antalya, Artvin, Aydın, Denizli, Bolu, İzmir, İstanbul, Kastamonu, Ordu [29,40].

Distribution in the world: Albania, Andorra, Armenia, Azerbaijan, Balearic Islands, Belarus, Belgium, Bosnia and Herzegovina, British Isles, Bulgaria, Corsica, Croatia, Cyprus, Czech Republic, Denmark, Estonia, Faroe Islands, Finland, France, Georgia, Germany, Greece, Jordan Netherlands Iceland, Iran, Iraq, Ireland, Israel, Italy, Latvia, Lithuania, Luxembourg, Macedonia, Moldova, Netherlands Norway, Russia, Romania, Russia, Russia, Sardinia, Sicily, Slovakia, Slovenia, Syria, the Arabian Peninsula, Turkey, Ukraine, Yugoslavia [51].

\section{Harpalinae}

Anissodactylus Dejean, 1829

Anisodactylus binotatus (Fabricius, 1787)

Material examined: Hatila National Park: 20.VI.2012, 2 $\widehat{\jmath} \widehat{\jmath}$, $q$; 14.VII.2013, 3 우, $\widehat{\jmath}$.

Distribution in Turkey: Adana, Anatolia (no locality), Kahramanmaraş [20,40].

Distribution in the world: Afghanistan, Albania, Algeria, Altai, Andorra, Armenia, Asor Islands, Austria, Azerbaijan, Bahrain, Belarus, Belgium, Bosnia and Herzegovina, Britain, Bulgaria, Caucasus, Crimea, Croatia, Cyprus, Czech Republic, Denmark, Estonia, Faroe Islands, Germany, Greece, Iceland, Ireland, Italy, Kazakhstan, Kyrgyzstan, Latvia, Lithuania, Luxembourg, Macedonia, Malta, Moldova, Montenegro, Morocco, Netherlands, North Africa, Norway, Poland, Portugal, Romania, Russia, Serbia, Siberia, Slovakia, Slovenia, Syria, Spain, Sweden, Switzerland, Tajikistan, Turkey, Turkmenistan, Ukraine, Uzbekistan $[1,6,37,47]$.

Ophonus Dejean, 1821

Ophonus (Hesperophonus) cribricollis (Dejean, 1829)

Material examined: Hatila National Park: 20.VI.2012, 2 ふ઼̊; 14.VII.2013, 3 우, ô. 
Distribution in Turkey: Anatolia (no locality), Ankara $[15,40]$.

Distribution in the world: Albania, Armenia, Austria, Bosnia and Herzegovina, Bulgaria, Croatia, Czech Republic, France, Georgia, Greece, Iraq, Iran, Spain, Israel, Italy, Macedonia, Moldova, Montenegro, Russia, Serbia, Slovakia, Switzerland, Turkey, Ukraine $[1,6,37,39,47]$.

Ophonus (Hesperophonus) azureus (Fabricius, 1775) Material examined: Hatila National Park: 20.VI.2012, 2

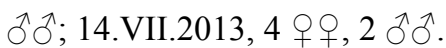

Distribution in Turkey: Anatolia, Ankara, Bayburt, Erzurum, Sinop, Trabzon [15,40,42].

Distribution in the world: Andorra, Bosnia and Herzegovina, Bulgaria, Croatia, Czech Republic, Denmark, France, Georgia, Germany, Greece, Hungary, Iraq, Iran, Italy, Kazakhstan, Kyrgyzstan, Latvia, Luxembourg, Macedonia, Moldova, Netherlands, Poland, Romania, Russia, Serbia, Slovakia, Slovenia, Spain, Sweden, Switzerland, Tajikistan, Turkmenistan, Ukraine, Uzbekistan $[1,6,37,39,47]$.

Ophonus (Hesperophonus) subquadratus (Dejean, 1829) Material examined: Hatila National Park: 20.VI.2012, 3

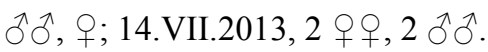

Distribution in Turkey: Anatolia (no locality), Antalya, Erzurum, İzmir [40,42,43].

Distribution in the world: Africa, Algeria, Bosnia and Herzegovina, Bulgaria, Caucasia, Croatia, Cyprus, France, Georgia, Greece, Iran, Israel, Italy, Libya, Malta, Moldova, Russia, Spain, Syria, Turkey, Turkmenistan, Ukraine $[6,37,39,47]$.

Turkey has a rich biological diversity in respect of geographical location. The scientific researches are being conducted for revealing the natural riches in Turkey. 32 species from Carabidae family were found and analyzed to determine the fauna of Carabidae Hatila National Park in Artvin (A.similata, A.saxicola, A.aenea, A.lucida, A.ovata, A.aulica, A.binotatus, B.elegans, B.crepitans, C.inquisitor, C.sycophanta, C.convexus, C.scabrosus, C.coriaceus, C.mulsantianus, C.graecus, H.affinis, H.smaragdinus, H.caspius, H.distinguendus, P.cupreus, P.rufipes, P.calceatus, Z.spinipes, D.halensis, C.axillaris, N.brevicollis, O.cribricollis, O.azureus, O.subquadratus, P.anthracinum, P.nigrita).

Calosoma sycophanta of Calosoma species from the subfamily of Carabinae is one of the most important species that was found in the area and its known that these species are predator life and used actively for biological contention. The species of the Zabrus spinipes and Pseudoophonus rufipes are from the fitofag species that cause the harmful effects in cultivated plants such as corn, wheat, and strawberry fields [52].

15 species were determined and accepted as the first record for the local fauna of Artvin at the end of the study (A.lucida, A.aulica, B.elegans, B.crepitans, C.scabrosus, A.binotatus, C.coriaceus, C.mulsantianus, C.graecus, H.affinis, H.caspius, O.cribricollis, O.azureus, O.subquadratus, P. anthracinum). Again in this study, totally ten species were accepted as the first record for the fauna of Eastern Black Sea (A.lucida, A.aulica, B.elegans, A.binotatus, C.coriaceus, C.mulsantianus, C.graecus, H.caspius, O.cribricollis, P.anthracinum).

When the evaluation is conducted based on the species it is clearly seen that the individuals of Harpalus smaragdinus are more comman than the others. The species of Harpalus distinguendus, Poecilus cupreus, Pseudoophonus calceatus, Zabrus spinipes, Dolichus halensis and Ophonus cribricollis have the lowest population in contrast with the other kinds.

When the insects in traps are evaluated, totally 864 insects were caught. 153 of them belong to the species of Amara and Brachinus, 378 of them belong to the species of Calosoma and Carabus, 172 of them belong to the species of Harpalus, Poecilus, Pseudoophonus and Zabrus, and finally 161 of them belong to Dolichus, Cymindis, Nebria, Ophonus and Pterostichus species. It is found that when we evaluate the feed amount in the traps we saw that live snails catch more than the traps have malt solution. Forestlands and cultivated areas are defined intense population of the species of Carabidae family $[8,53]$. Hatila National Park was exposed to the attacks bark beetles in previous years. There are many trees that broken and ruined in this park area. These trees enhance the insect diversity in the area. It is possible to increase the number of species if this study is applied provincial in Hatila National Park. In conclusion, it is based on this research that 32 species of Carabidae family in Hatila National Park is a sign for the richness of species in the region.

\section{ACKNOWLEDGEMENT}

This study was performed as a M.Sc. thesis by the second author and accepted by the Graduate Schol of Natural and Applied Sciences, Artvin Coruh University, Artvin, Turkey.

\section{REFERENCES}

[1] Hurka, K. (1996) Carabidae of the Czech and Slovak Republics. Print Centrum, a. s., Zlin, 565pp.

[2] Larochelle, A. (1990) The food of carabid beetles (Coleoptera; Carabidae, including Cicindelinae). Association of Amateur Entomologist of Quebec, Fabreries, supplement. 5:1-132pp. 
[3] Thiele, H.U. (1977) Carabid Beetles in Their Environments. Springer-Verlag, Berlin, 369 pp.

[4] Stork, N. D. (1990) The role of ground beetles in ecological and environmental studies. Andover: Intercept, $424 \mathrm{pp}$.

[5] Larsen, J. K., Work, T.T. \& Purrington, F.F. (2003) Habitat use patterns by ground beetles (Coleoptera: Carabidae) of northeastern Lowa. Pedobiologia, 47: 288-299.

[6] Löbl, I. \& Smetana, A. (2003) Catalogue of Palaertic Coleoptera. Volume I. Archostemata-MyxophagaAdephaga. Apollo Books. Stenstrup, Denmark, 819 pp.

[7] Kromp, B. (1999) Carabid beetles in sustainable agriculture: a review on pest control efficacy, cultivation impacts and enhancement. Agric. Ecosyst. Environ., 74: 187- 228.

[8] Kromp, B. (1989) Carabid beetle communities (Carabidae: Coleoptera) in biologically and conventionally farmed agroecosystems. Agric. Ecosyst. Environ., 27: 241-251.

[9] Luff, M. L., Eyre, M.D. \& Rushton, S.P. (1992) Classification and prediction of grassland habitats using ground beetles (Coleoptera, Carabidae). J. Environ. Management, 35: 301-315.

[10] Duelli, P. \& Obrist, M.K. (1998) In search of the best correlates for local organismal biodiversity in cultivated areas. Biodivers. Conserv. 7: 297-309.

[11]Rainio, J. \& Niemelä, J. (2003) Ground beetles (Coleoptera: Carabidae) as bioindicators. Biodiversity and Conservation, 12: 487-506.

[12]Lodos, N. (1983) Türkiye faunasına ait ekin kambur böcekleri, Zabrus Clairv.(Coleoptera: Carabidae) cinsinin yeniden gözden geçirilmesi. Türk Bit. Kor. Derg., 7: 51-63.

[13] Casale, A., Turin, H. \& Penev, L. (2003) The Genus Carabus in Europe, Pensoft, 511pp.

[14] Kesdek, M. \& Yıldırım, E. (2008) Contribution to the knowledge of Carabidae fauna of Turkey. Part 7: Pterostichini (Coleoptera, Carabidae, Pterostichinae). Linzer biol. Beitr. 40:1, 813-816.

[15] Kocatepe, N. \& Mergen, O. (2004) Ankara ili Carabidae (Coleoptera) familyası türleri üzerinde faunistik araştırmalar. Türk Entomol. Derg., 28:4, 295-309.

[16] Schweiger, H. (1962) Neue und wenig bekannte Carabus-Formen aus der Turkei, (Coleoptera, Carabidae). İstanbul Üniv. Fen Fak. Mec., 27: 159167.

[17] Schweiger, H. (1962) Neue und wening bekannte Carabiden aus kleinasien (Ergebnisse Der Österreichisch- Türkischen Anatolienexpeditionen), İstanbul Üniv. Fen Fak. Mec., 28: 137-154.
[18] Vigna Taglianti, A. (1973) Considerazioni sui Carabidi cavernicoli Ed Endogei Dell'Asia Minore (Coleoptera, Carabidae), Int. J. Speleol., 5: 349- 360.

[19]Erçelik, M. (1975)Evolusiyon Merkezi Uludağ'ın Coleopter sistematiği ve bazı türlerin ekolojisi. İstanbul Üniversitesi Fen Fakültesi Monografileri, $108 \mathrm{~s}$.

[20] Avgin, S.S. \& Prunier, D. (2010) Contribution to the taxonomy of the Genus Cychrus Fabricius, 1794 (Coleoptera: Carabidae) from Turkey and their relations with Cychrus species from Balkans to Caucasus. Türk. Entomol. Derg., 34:3, 307-322.

[21] Yücel, E. \& Sahin, Y. (1988) Eskişehir ve Afyon yöresi bazı Carabidae (Coleoptera) türlerinin morfolojisi ve ekolojisi üzerine çalışmalar I. Anadolu Üniv. Fen Edeb. Fak. Derg., 25-29.

[22] Ledoux, G. \& Roux, P. (1990) Le genre Nebria (Coléoptérés, Nebriidae). I - Redéfinition de Sous Genres Alpaeus et Nebria, Description de Sept Especes et d'une Sous-Espece Nouvelles de Turquie. L'Entomologiste, 46: 65-97.

[23] Turktan, H. (1998) Eskişehir Çevresi Carabidae (Insecta: Coleoptera) Üzerine Faunistik Araştırmalar. Anadolu Üniversitesi Fen Bilimleri Enstitüsü ,Yüksek Lisans Tezi, Eskişehir, 34 p.

[24]Donabauer, M. (2004) Sechs neue arten der gattung Trechus Clairville, 1806 aus der Nord-Türkei (Coleoptera: Carabidae). Z. Arb. Gem. Öst. Ent., 56: 43-60.

[25] Kesdek, M. (1998) Erzurum İli Harpalini Tribüsü (Coleoptera, Carabidae, Harpalinae) Türleri Üzerinde Faunistik ve Sistematik Çalışmalar. Atatürk Üniversitesi Fen Bilimleri Enstitüsü, Yüksek Lisans Tezi, Erzurum, 53s.

[26] Battoni, F. (1984) Revisione di un gruppo di Calathus bon dell'Anatolia con descrizione di due nuove specie (Coleoptera Carabidae). Bull. Soc. Ent. Ital., 116 (13): 17-26.

[27] Avgin, S.S. \& Cavazzuti, P. (2011) The studies made on Turkish Carabinae with checklist and bibliography (Coleoptera: Carabidae). Turk J. Zool., 35:3, 403-432.

[28] Kesdek, M. \& Yıldırım, E. (2007) Contribution to the knowledge of the Carabidae fauna of Turkey Part 4: Dryptini, Lebiini and Zuphiini (Coleoptera: Carabidae, Lebiinae). Band 28, Heft 22:277-284 ISSN 0250-4413.

[29] Tezcan, S., Jeannel, C., Keskin, B., (2007) Ground beetles (Coleoptera: Caraboidea) of the ecologically managed cherry orchards of Western Anatolia (Turkey) along with some new additional data, Anadolu Üniversitesi Bilim ve Teknoloji Dergisi, 8:1, 53-63. 
[30] Aslan, B., Aslan, E.G., Karaca, I. \& Kaya, M. (2008) Kasnak Meşesi Tabiatı Koruma Alanında (Isparta) farklı habitatlarda çukur tuzak yöntemi ile yakalanan Carabidae ve Tenebrionidae (Coleoptera) türleri ile biyolojik çeşitlilik parametrelerinin karşılaştırması, SDÜ Fen Edebiyat Fakültesi, Fen Dergisi (E-Dergi), 3:2, 122-132.

[31] Avgin, S.S. (2006) Distribution and diversity of ground beetles in Baskonus Mountain National Park of Turkey. Journal of Environmental Biology. 27:3, 515-521.

[32] Kesdek, M. (2012) A Contribution to the knowledge of the Carabidae (Coleoptera) fauna of Turkey. Acta Biol. Univ. Daugavp. 12 (1).

[33] Kesdek, M. \& Yildırım E. (2013) Contributıons to the knowledge of the Genus Harpalus Latreille, 1802 fauna of Turkey (Coleoptera: Carabidae: Harpalınae). Mun. Ent. Zool. Vol. 8, No. 1.

[34] Ansin, R., Ozkan, Z.C. \& Eminagaoglu, O. (2000) Artvin-Atila (Hatilla) Vadisi Milli Parkı'nın vejetasyon yapısına genel bir bakış. Journal of Artvin Forestry Faculty of Kafkas University, 1:1, 59-71.

[35]Ceylan, S. (1995) Artvin Yöresinin Coğrafi Etüdü, Atatürk Üniversitesi Sosyal Bilimler Enstitüsü, Doktora Tezi, Erzurum.

[36] Lindroth, C.H. (1974) Handbooks for the identification of British insects, vol IV, Part 2. Royal Entomological Society of London, $149 \mathrm{p}$.

[37] Gueorguiev, V. B. \& Gueorgiev, B.V. (1995) Catalogue of the ground-beetles of Bulgaria (Coleoptera: Carabidae). PENSOFT Publishers, SofiaMoscow, 279 pp.

[38] Forsythe, T. G. (1987) Common ground beetles. Richmond Publishing Co. Ltd., London, 57pp.

[39] Kryzhanovskij, O. L., Belousov, I.A., Kabak, I.I., Kataev, B.M., Makarov, K.V. \& Shilenkov, V.G. (1995) A checklist of the ground-beetles of Russia and Adjacent Lands (Insecta, Coleoptera, Carabidae). Sofia, Bulgaria. Moscow, $271 \mathrm{pp}$.

[40] Casale, A. \& Vigna Taglianti, A. (1999) Caraboid beetles (excl. Cicindelidae) of Anatolia, and their biogeographical significance (Coleoptera, Caraboidea). Biogeographia, Lavori della Società Italiana di biogeografia, Siena, Italy, 20: 277-406.

[41] Neculiseanu, Z.Z. \& Matalin, A.V. (2000) A catologue of the ground-beetles of the republic of Moldova (Insecta, Coleoptera: Carabidae). Sofia, Bulgaria, 245p.

[42] Kesdek, M. \& Yıldırım, E. (2003) Contribution to the knowledge of Carabidae fauna of Turkey Part 1: Harpalini (Coleoptera, Carabidae, Harpalinae). Linzer Biol. Beitr., 35:2, 1147-1157.
[43] Oncuer, C. (1991) A catalogues of the parasites and predators of insect pest of Turkey. Ege University, Faculty of Agriculture Publication, İzmir, 354pp.

[44] Kesdek, M. (2007) Kuzeydoğu Anadolu Bölgesi Pterostichinae (Coleoptera: Carabidae) Türleri üzerinde Faunistik ve Sistematik Çalışmalar, Atatürk Üniversitesi Fen Bilimleri Enstitüsü, Doktora Tezi 239s.

[45] Kesdek, M. \& Yıldırım, E. (2010) Contribution to the knowledge of Carabidae fauna of Turkey Part 8: Amarini (Coleoptera: Carabidae, Pterostichinae). Band 31, Heft : ISSN 0250-4413 Ansfelden, 4.

[46] Tanyeri, R. (2011) Aspat (Muğla) Yöresinde Bulunan Carabidae, Tenebrionıdae ve Staphylınıdae (Coleoptera) Familyalarına Bağlı Türler Üzerinde Faunistik Çalışmalar, Ege Üniversitesi Fen Bilimleri Enstitüsü, Biyoloji Anabilim Dalı, Yüksek Lisans Tezi, Balıkesir.

[47] Trautner, J. \& Geigenmüller, K. (1987) Tiger Beetles Groud Beetles, Illustrated Key to The Cicindelidae and Carabidae of Europe. Josef Margraf Publisher. Gaimersheim, Germany, 488 pp.

[48] Kocatepe, N. (2011) Orta ve Doğu Karadeniz Bölgesi Carabidae (Coleoptera) Familyası Üzerinde Sistematik Çalışmalar. Hacettepe Üniversitesi Fen Bilimleri Enstitüsü. Biyoloji Anabilim Dalı, Doktora Tezi, Ankara.

[49] Göktürk, T. (2002) Artvin İlinde İğne Yapraklı Orman Ağaçlarında Yaşayan Coleoptera Türleri İle Predatör Ve Parazitoidleri. Karadeniz Teknik Üniversitesi Fen Bilimleri Enstitüsü Doktora Tezi, 269s.

[50] Surgut, H. (2011) Karabiga (Çanakkale) Yöresinin Carabıdae, Tenebrionıdae, Elaterıdae, Silphidae ve Staphylınıdae (Coleoptera) Türlerinin Çukur Tuzak Yöntemiyle Belirlenmesi. Balıkesir Üniversitesi Fen Bilimleri Enstitüsü. Biyoloji Anabilim Dalı, Yüksek Lisans Tezi, Balıkesir.

[51] Anonymous, (2016) Fauna Europae, www.faunaeur.org. 18. 12.2016.

[52] Lodos, N. (1989) Türkiye Entomolojisi IV (Kısım 1), Ege Üniversitesi Ziraat Fakültesi Ofset Basımevi, Bornova- İzmir.

[53] Varvara, M. (1991) Andriescu, I. Comparative analysis of the Carabidocoensis from the "Valen Lui David" Phyto-and agrerocoenotic comlex (Iasi). Proceedings of the 4th ECE/XII. SIEEC. 